\title{
Students' Attitudes to Shared Decision Making
}

Author: Tim Harrison. Manchester Pharmacy School, The University of Manchester

Background: Shared decision making (SDM) is considered a basic requirement for patient care and the ability for undergraduate pharmacy students to demonstrate competency in this area is a GPhC requirement. This exploratory study looked at two interventions to assess the attitudes of first year pharmacy students to SDM.

Method: The interventions were organised early in semester 1 of year 1 in 2013/2014. The first intervention was to re-design four communication lectures, to focus on patient-centred communication, and the use of videos, from HealthTalkOnline ${ }^{1}$. The videos depicted patients discussing their experiences and perspectives of communication with healthcare professionals. The second intervention was a patient-led workshop, focusing on the patients' perspective of managing their health needs. To evaluate the interventions a validated instrument, the updated Leeds Attitude to Concordance Scale (LATConlI), was used, with students answering the questions using TurningPoint $\AA$, before the first lecture (T1), after the fourth lecture (T2) and after the workshop (T3).

Results: Of $1541^{\text {st }}$ year students, 68, 39 and 16 responded at T1, T2 and T3 respectively, answering all of the 20 LATConll questions. A positive shift in attitude was seen between $\mathrm{T} 1$ and $\mathrm{T} 2$, from 37.5 to 43.4 out of 60 , however the small response rate at T3 made detection of a further shift in attitudes meaningless.

Discussion: The shift in attitude at T2 was encouraging, however, the use of the LATConll instrument via TurningPoint $\circledast$ introduced some limitations, as students must answer all of the questions for the results to be valid, the process was time consuming and the reason for non-response to questions was not apparent. Introduction of a neutral answer into the LATConll instrument may increase response rate, but would mean that results could not be compared with other studies.

\section{References}

1.HealthTalkOnline.org [Internet]. Oxford: HealthTalkOnline; 2014 [updated 2014; cited 2014 April 1]. Availlble from: http://www.healthtalkonline.org

2.Knapp P; Raynor DK; Thistlethwaite JE; Jones MB. A questionnaire to measure health practitioners attitudes to partnership in medicine taking: LATCon II.Health Expectations: An International Journal Of Public Participation In Health Care And Health Policy [Health Expect]. 2009 Jun; Vol. 12 (2), pp. 175-86 